\title{
INTERNET ADDICTION: A REVIEW OF THE FIRST TWENTY YEARS
}

\author{
Martin Mihajlov $^{1}$ \& Lucija Vejmelka ${ }^{2}$ \\ ${ }^{I}$ Cyril and Methodius University, Faculty of Economics, Skopje, Macedonia \\ ${ }^{2}$ University of Zagreb, Faculty of Law, Department of Social Work, Zagreb, Croatia
}

received: 9.3.2017;

revised: 3.5.2017;

accepted: 12.7 .2017

\begin{abstract}
SUMMARY
Easy access to communication and information technologies has increased our dependence on technology for various aspects of our lives. Nevertheless, this remarkable growth of Internet Usage has been inextricably paired with a rise of excessive and dysfunctional Internet use. Conceptualized around 1996, a few years after the inception of the World Wide Web, Internet addiction has developed into a global issue influencing varying segments of the population at different levels. Despite heated debates on its addictive nature, consensus is emerging regarding the existence of this problematic behavior.

In this paper we provide a comprehensive overview of the literature on Internet addiction in last 20 years. Purpose of this paper is to present crucial findings on Internet addiction to health profession.

Besides numerous benefits of Internet use, the virtual environment brings various risks in every age group. The Internet is very significant in the everyday activities of children and youth and professional interventions with this age group should be specific considering their developmental characteristics. Exposure to online risks can have long-lasting and intense negative effects. Effective programs in prevention and treatment should include a multi-sectoral and interdisciplinary approach. Detail review of the symptomatology, diagnosis model an possibilities of treatment can be multiple beneficial to the health professionals and other helping professions due to actual needs for interventions in the field of the internet addiction treatment.

Internet addiction is slowly becoming a societal concern as it particularly affects adolescents and children, who are more exposed and consequently more vulnerable. Findings presented in the paper can benefit in practice of treatment internet addiction and also as framework for further researches in the field.
\end{abstract}

Key words: Internet addiction - prevalence - symptomatology - diagnosis model - treatment

$* * * * *$

\section{INTRODUCTION}

Along with new technologies, the Internet has reshaped and improved many aspects of our lives by being integrated in the daily experience. Internet has become more available, offers more services and its usage is growing in every age group. Approximately $46 \%$ of the world population is on the Internet today, an incredible growth compared to only less than 1\% in 1995 (Internet Live Stats). The world's most developed countries already have an Internet penetration level over $90 \%$, for example Iceland, 100\%, Norway, 98\%, Denmark, 96.3\%, UK, $92.6 \%$, or Japan, $91.1 \%$, while the highest growth rates are recorded on the African continent in developing countries like Mali, $18.6 \%$, Cameroon, $16.5 \%$, or Côte d'Ivoire, $14.3 \%$. Overall rate in Europe is $73,5 \%$ of and Croatia with $75 \%$ of internet users (Internet World Stats 2017). Specific of internet use in Croatia implies that Internet is still ,mostly sporadically and very unevenly distributed among different regions which imply there are significant differences in internet usage among children and young people“ (Kanizaj 2016). Especially over the past decade, advances in mobile and wearable technologies have allowed a seamless and ubiquitous connection of individuals to the online world, thus being the key drivers of a phenomenon called Internet Addiction - IA (Turel \& Serenko 2010).
Considering that the Internet is an integral part of social and professional life, it is necessary to understand the fundamental reasons for its use. Technology brings various benefits in everyday life including gathering information, easier communication and learning in general. Studies in the field highlight that internet use can help improve results on tests and increase motivation for learning among young people (Guan \& Subrahmanyam 2009). Also, perceived quality of life can be affected in the absence of Internet access (Pontes et al. 2015).

While the use of technology is considered as a positive phenomenon, recent empirical evidence reveals that technology addiction augments a user's intrinsic and extrinsic gain perceptions about a system, leading to system overuse at an unhealthy elevated level (Turel et al. 2011). Addiction symptoms prevalent among technology addicts can cause negative personal, societal and workplace related ramifications (Charlton \& Danforth 2007), thus advocating the question of how technology use can be adjusted to healthy levels.

Excessive Internet use is seen as a form of technological addiction which touches a large scope of behavioral responses (Marks 1990). Viewed through the prism of addiction, IA is a relatively recent and fast growing clinical phenomenon (Saville et al. 2010). Due to its fast growth the attitude of many countries towards Internet addiction is as a potential threat to public 
health. For example, China has reported that approximately 10 million citizens have scored high on Internet addiction tests (Block 2008). Despite this growth, there are still many disagreements in the field ranging from terminology and diagnosis to treatment. Researchers even disagree with the concept of Internet addiction as a distinct disorder, emphasizing instead that the Internet is a medium, not a substance and hence a manifestation of other disorders like ADHD, depression, social loneliness and anxiety (Recupero 2010).

Purpose of this paper is to present extent literature review on internet addiction of last two decades, from the pioneer researches to recent studies around the world. In the article we will define terminology of IA and then discuss operationalization of the IA through detail description of available measures. Data about global prevalence of IA is also included. Causality and diagnosis models including symptoms and the treatment described from different perspectives presented in the article is important for health professionals especially ones who works with children and youth. Relevance of the topic is confirmed by actual trends in growing internet especially in the age group of children and youth usage (Croatian Bureau of Statistics 2016) and a fact that Croatian medical institutions started to implement programs of treatment Internet addiction.

\section{INTERNET ADDICTION TERMINOLOGY}

In the US, a serious research pioneer in the field is Dr Kimberley Young who first published a case study regarding a 43-year old woman addicted to email (Young 1996). This was followed by the first seminal Internet addiction study (Young 1998b) which collected approximately 600 cases of people who suffered problems in their everyday life offline because they were unable to control Internet use. The research into Internet addiction in Europe was initiated by Griffiths's paper on technological addictions (Griffiths 1995), followed by a general paper on Internet addiction (Griffiths 1996a) and specific aspects such as Internet gambling addiction (Griffiths 1996b).

The term "addiction" has been criticized heavily by peer scientists which has allowed for the emergence of less controversial terminologies describing the same phenomenon (Pezoa-Jares \& Espinoza-Luna 2013). Currently, there is no consensus for a consistent nomenclature with some of the different proposed terms being: Internet dependency (teWildt 2011), pathological Internet use (Morahan 2000), problematic Internet use (Davis et al. 2002), compulsive computer use (Black et al. 1999), etc. On the other hand, the reference to the Internet as an addictive medium is not suitable considering that many of the excessive Internet users are not Internet addicts, but use the Internet as a medium to fuel their specific addictions (Griffiths 1999). Therefore, there have been several different proposals about IA classifications.
Based on empirical data, Young et al. (1999) view IA as an umbrella term for a wide variety of behaviors and impulse control problems that can be divided into five subtypes:

- cybersexual addiction - compulsive use of adult websites for cybersex and cyberporn;

- cyber-relationship addiction - over-involvement in online relationships;

- net compulsions - obsessive online gambling, shopping or day-trading;

- information overload - compulsive web surfing or database searches;

- computer addiction - obsessive computer game playing.

Alternatively, Davis (2001) proposes a theoretical cognitive and behavioral model of pathological Internet use (PIU). The model differentiates between addictions to the Internet versus addictions on the Internet through the following two categorizations:

- Generalized Problematic Internet Use (GPIU) - a multidimensional overuse of the Internet itself not concerned with any specific online activity;

- Specific Problematic Internet Use (SPIU) - pathological indulgence into an online behavior through a specific function and/or application.

With this classification GPIU can be considered as an addiction to the Internet, while SPIU can be considered as addictions on the Internet (Griffiths \& Pontes 2014).

Essentially, IA research before 2000 was heavily focused on general Internet use. The trend has shifted and the Internet is now more often treated as a medium for numerous and independently existing activities. When it comes to the cause of addiction, this approach implies that online content and online activities take precedence over the medium itself. However, as the medium itself changes the essential aspects of the original activity, both content/activity and medium become essential ingredients of IA (Király et al. 2014).

The use of the Internet brings various benefits to various aspects of everyday life; it facilitates business and increase productivity, it is used for a spectrum of educational purposes and it enables easier communication. Research shows that educational activities as school homework, searching for useful information or searching for medical information is not related with Internet addiction (Eijnden \& Meerkerk 2008, Vejmelka et al. 2017). In addition, Tsitsika \& Janikian (2014) came to the conclusion that adolescents that use internet for educational and research purposes are less likely to develop addiction.

As a matter of fact, after it initial conceptualization, it is the rapid evolvement of online applications that has changed the view of IA. Functional activities such as social interaction, role-playing games, gambling, or pornography have replaced the early manifestations of potentially addictive activities, namely email, chatting and texting. In its fifth review, the Diagnostic and Statistical 
Manual of Mental Disorders DSM-5 included Internet Gaming Disorder (IGD) as a supplement which indicates that IDG has been recognized as a condition that requires specific attention from professionals. It requires more clinical research and experience before being included as a formal disorder (Petry et al. 2015). Nevertheless, IGD is the only Internet-related condition officially included in a diagnostic manual (APA 2013). In IGD, a gaming addict is viewed as someone that uses the Internet as a medium in order to addictively engage in gaming. This leads to conceptualizing Internet-related disorders, such as IGD, online social networking addiction or online sexual addiction as a spectrum of behavioral addictions (Billieux 2012). In an extensive literature research review on Internet addiction and pornography addiction, Love et al. (2015) concludes that Internet pornography addiction fits into the addiction framework and shares similar basic mechanisms with substance addiction. It stimulates the brain's reward system, and the person is searching for additional excitement online. The excessive use of internet pornography could also be explained from a neuro-biological aspect, as there is an expectation of a more intense content the individual seeks further excitement by increasing frequency of online sexual activities (Hilton 2013, Spicer et al. 2007).

It is impossible to deny that professional practice in the health sector and helping professions are recognizing and carrying out interventions with online behavioral problems. Furthermore, evidence based practice is significant for Internet addiction difficulties suggesting the importance of future research in this field. Although related by the Internet medium, these and similar disorders need to be viewed as independently dysfunctional behaviors. Tentatively, it has been indicated that the term "Internet addiction" in general should be actively replaced by the respective addiction to a specific online activity when the behavioral addiction patterns for that activity are ascertained (Starcevic 2013). With this in consideration, Cantelmi et al. (2000), proposed the term internet-related psychopathology (IRP) as the clinical conditions surrounding IA are not an internet dependency, but a group of specific psychopathological situations in an online environment. Other suggested terms for the disorder are "internet-mediated psychopathology" (Tonioni 2013) and "internet spectrum dependency" (Karaiskos et al. 2010).

For consistency, from the various terminologies used to define this phenomenon in this paper we will use the term "Internet addiction" to describe the pathological state of overindulging in Internet-related activities.

\section{MEASURING INTERNET ADDICTION}

Since the inception of the field, a myriad of instruments have been developed to assess IA among a target population. However, most instruments have not been verified and their psychometric properties have not been investigated within the context of different user groups or culture. As a matter of fact, in the most exhaustive literature review to date, Laconi et al. (2014) have identified 45 tools which measure and assess Internet addiction through either scales, interviews or diagnostic criteria. All developed instruments are self-report questionnaires constructed using a theoretical basis and have no clinical validation. Length-wise the questionnaires are relatively short, ranging from 8 to 36 items on either dichotomous or dimensional scales, and are hence easy to administer in both research and clinical environments. Only some instruments have reported criteria and/or cutoff points for IA, although they are seldom based on empirical analyses. From all of these tools only 17 had their psychometric properties evaluated more than once, and only 10 had three or more evaluations. This implies that most of the reported scales require further examination before they can be used by researchers and/or clinicians as measures for assessment of IA. The validity of these scales is questionable mostly due to the lack of definition for IA and its current grounding in multiple theoretical frameworks (Beard 2005, Weinstein \& Lejoyeux 2010).

Considering that there are several exhaustive systematic reviews of IA instruments (Lortie \& Guitton 2013, Laconi et al. 2014), in this section we present a selective review of the most frequently used and wellresearched instruments. For these instruments we discuss their psychometric properties by reporting on the findings for reliability, validity, and factor structure.

\section{Instruments for IA evaluation}

The Internet Addiction Test (Young 1998a) is based on the Internet Addiction Diagnostic Questionnaire IADQ (Young 1998b) and assesses IA based on criteria for the diagnosis of pathological gambling. The test consists of 20 self-reported items scored on a 5-point Likert-type scale ranging from "rarely" to "always" allowing for a dimensional rather than categorical assessment. The items include concepts such as loss of control, neglecting everyday life, behavioral and cognitive salience, negative consequences, mood modification, and deception. Based on these items users can be categorized as normal, with frequent problems or with significant problems, although the cutoff scores, 40/70, can be considered as arbitrary as they are not based on empirical considerations. Different cut-off criteria used in researches significantly reduces the possibility of results comparison and should be considered. The modified IAT with a 6-point scale includes the "does not apply" option (Young 2010) and differentiates between normal users and users with mild, moderate or severe Internet addiction with cutoff scores set at $31 / 50 / 80$. Several studies have shown an excellent testretest reliability, $r>=0.75$, and excellent internal consistency, a $=0.92$ (Barke et al. 2012, Lee et al. 2013, Osada 2013). Psychometric validations of the IAT have consistently resulted in differing dimensional structures, mostly dependent on culture, which strongly suggests that sociocultural factors impact Internet addiction assessment (Widyanto et al. 2011). 
The Compulsive Internet Use Scale - CIUS (Meerkerk et al. 2009) was developed primarily on criteria for pathological gambling. It originally included 14 items with a 5-point Likert-type scale, ranging from "never" to "very often". However, it has also been validated in a 9-item form for adolescents (Cartierre et al. 2011) and it exists in a 17-item form (Aa et al. 2008). Three cut-off scores have been proposed: 18, 21, and 37 (Guertler et al. 2014). The CIUS revealed good split-half reliability, $\mathrm{r}=0.89$, good convergent validity with time spent online, $0.33<\mathrm{r}<0.68$, excellent test-retest reliability, $\mathrm{r}=0.83$, and excellent internal consistency, $\mathrm{a}=0.90$ (Alavi et al. 2011; Khazaal et al. 2011). Several factor analysis studies suggest a single dimension (Peukert et al. 2012, Wartberg et al. 2014, Dhir \& Helsinki 2015), mostly referring to "compulsive and impulse control elements".

The Chen Internet Addiction Scale - CIAS (Chen et al. 2003) is another questionnaire developed on criteria for pathological gambling and substance dependence. The test consists of 26 self-reported items scored on a 4point Likert-type scale ranging from "very unlike me" to "very like me". The test evaluates the core symptoms of addiction, such as tolerance, compulsive use, and withdrawal and related symptoms such as negative impact on social activities, interpersonal relationships, physical condition, and time management. It has good psychometric properties with suggested cut-off points at 58 for screening and 64 for diagnosis of Internet addiction that have a high sensitivity with a diagnostic accuracy with $87.6 \%$ (Ko et al. 2005). The instrument has a good test-retest reliability, $\mathrm{r}=0.85$, and excellent internal consistency for the respective subscales, $\alpha=0.93$ (Kesici \& Sahin 2010). Psychometric properties are determined as a 5-factor solution, including "compulsion", "withdrawal", "tolerance", "time management" and "interpersonal and health problems" (Ramezani et al. 2012).

The Online Cognition Scale - OCS (Davis et al. 2002) is a questionnaire based on cognitive-behavioral theory. The questionnaire consists of 36 items scored on a 7point Likert-type scale ranging from "strongly disagree" to "strongly agree". It has shown excellent test-retest reliability, $\mathrm{r}=0.9$, excellent internal consistency, and good construct validity $0.47<\mathrm{r}<0.81$ (Ozcan \& Buzlu 2005, Song 2007). Due to its interpretive nature, the scale has no official cut off points for categorization of addiction levels. Factor analysis defines a 4-factor solution consisting of "social comfort", "loneliness", "diminished impulse control" and "distraction" (Zec 2005).

The Generalized Problematic Internet Use Scale GPIUS (Caplan 2002) is another questionnaire based on cognitive-behavioral theory. Initially, it consisted of 29 items scored on a 8-point Likert-type scale with a good test-retest reliability, $r=0.73$, and good split-half reliability. The second version of the questionnaire, GPIUS-2 is composed of 15 items also scored on an 8-point Likert-type scale ranging from "strongly disagree" to "strongly agree". The updated scale has a good convergent validity with time spent online, $\mathrm{r}=0.44$, and depression, $\mathrm{r}=0.27$ (Gámez-Guadix et al. 2012). Psychometric evaluations reveal 6 and 7 factor solutions for the GPIUS (Caplan 2002) and 4-factor solutions for the GPIUS-2 (Fioravanti et al. 2013).

Examples of other IA instruments include Problematic Internet Use Questionnaire - PIUQ (Demetrovics et al. 2008), Problematic Internet Use Scale - PIUS (Morahan 2000), Computer and Internet Use - CIU \& CIU-2 (Pratarelli et al. 1999; Pratarelli \& Browne, 2002), Problematic Internet Use Diagnostic-Interview PIUD-I (Beard \& Wolf 2001) and others.

Instead of continuing to develop new instruments, the psychometric properties of current tools need to be investigated in more detail in order to enable IA research to move towards developing a 'gold standard' for assessment (Wartberg et al. 2014). Furthermore, the process of psychometric property assessment itself needs to be unified as different researchers have adopted different techniques for validating IA instruments. This approach will effectively enable the comparison and synthesis of results between different tools.

\section{GLOBAL PREVALENCE OF IA}

With the heterogeneity of assessment instruments and varying samples, comparing the prevalence of IA across any demographic characteristic is rather difficult, especially since most studies use questionnaires similar to the Internet Addiction Test applied through online recruitment, instead of comprehensive clinical interviews in a random general population sample (Kuss et al. 2014). Hence, the numbers presented in this section have only a provisional significance, as consistent diagnostic criteria, representative offline samples and validated questionnaires are needed to determine accurate and comparable IA rates.

International prevalence rates have a great variance depending on measurement method and target population. A worldwide review carried on behalf of the German health ministry reports that international prevalence rates for IA range from $1.5 \%$ to $8.2 \%$ (Petersen et al. 2009). Another major cross-sectional survey of high school students in 11 European countries, with an average age of 14.9 years, revealed an overall prevalence rate of 4.4\% (Durkee et al. 2012). A more detailed overview is presented in Table 1.

Compared to the rest of the world, Asian cultures have had the most significant problems with IA (Aboujaoude et al. 2006). Reported rates vary from $2.4 \%$ to $13.5 \%$ in Chinese schools (Deng et al. 2007; $\mathrm{Wu}$ et al. 2013) and from $1.6 \%$ to $20.3 \%$ in South Korean adolescents (Kim et al. 2006). In the United States, rates vary from $0.7 \%$ to $26.3 \%$ in adolescents and college students (Moreno et al. 2011). In Europe, rates have a similarly broad range between $1 \%$ and $18.3 \%$ in random surveys (Bakken et al. 2009; Rumpf et al. 2014). Studies of IA rates in other countries also fluctuate between $0.7 \%$ in Indian college students (Goel et al. 2013) to $17.3 \%$ in Qatari adolescents and young 
adults (Bener \& Bhugra 2013). Recent study in Croatia shows prevalence rate of $3.4 \%$ on high school students (Vejmelka et al. 2017). At this moment the cultural differences cannot be explained. This possibly relates to a previosly raised issue about different FA results. Without a unified instrument or clinical approach to diagnosing IA it producing a correct interpretation is highly unlikely.

Table 1. World-wide prevalence of Internet addiction

\begin{tabular}{llc}
\hline Country & Age group & Prevalence rates \\
\hline United States & Adult & $0.7-6 \%$ \\
& College & $4-25 \%$ \\
Greece & Adolescents & $3.1-15.3 \%$ \\
Italy & Adolescents & $5.4 \%$ \\
& College & $5 \%$ \\
Norway & General & $1 \%$ \\
United Kingdom & College & $18.3 \%$ \\
India & Adolescents & $0.7 \%$ \\
& College & $0.7 \%$ \\
Qatar & College & $17.3 \%$ \\
South Korea & Adolescents & $1.6-20.3 \%$ \\
China & Middle school & $2.4-6.3 \%$ \\
& Adolescents & $5.5-13.5 \%$ \\
Taiwan & College & $6.4 \%$ \\
Croatia & College & $17.9 \%$ \\
\hline
\end{tabular}

\section{DIAGNOSIS AND SYMPTOMS OF INTERNET ADDICTION}

Addictions can be discerned by both psychological and physical characteristics. Physical characteristics, typical for substance abuse addictions, are manifested by compulsive consumption due to the need to eliminate anxiety brought on due to the absence of the said substance. Psychological characteristics are most noticeable during withdrawal and represent the behavioral issues due to psychological dependence. Considering that IA is a behavioral addiction, to understand the clinical nature of overuse and its diagnosis it is necessary to focus on the psychological issues.

\section{Causes}

Compulsive Internet use provides a psychological escape mechanism to avoid real or perceived problems. Considering that addictive personalities are more likely to suffer from negative thinking (Hall \& Parsons 2001), which in turn leads to low self-esteem and pessimistic attitudes, the anonymous interaction over the Internet alleviates these self-imposed inadequacies. This is especially evident in young people who 20-years ago used poetry, music, and sports to express their needs and feelings, whereas now they turn to online activities to compensate their identity (Tao 2005). This escapism leads a person to depart from an unpleasant reality in order to create a virtual "ideal self" liberated from real-life stress and limitations ( $\mathrm{Li}$ et al. 2011). Hence, the motivation for going online and spending excessive time have been explored in context of specific online activities and content as it relates to the particular individual.

Escapism is most evident in online gaming, where researchers have investigated it relationship with IA by exploring interaction effects between psychosocial problems and alleviating motivations for use. The anonymity in online environments people allows highly anxious individuals to compensate their loneliness by engaging in socialization through mutual gameplay. KardefeltWinther (2014) showed that the relationship between stress and online gaming is mediated by the motivation for escapism. Similar findings have been especially observed among players of massive multiplayer online role-playing games (Zanetta-Dauriat et al. 2011, Lemenager et al. 2013). These findings suggest it is necessary to further explore motivations for gaming and psychosocial well-being in conjunction.

From the perspective of social media, expanding social networks to establish or enhance relationships is viewed as an approach to improving self-confidence, social abilities, and social support (Smahel et al. 2012). Individuals with heightened social anxiety, may perceive this form of communication a more acceptable form of interaction, due to the greater degree of control over one's image and the lower risk of negative evaluation, which in turn may lead to IA (Lee \& Stapinski, 2012). The online environment appeals to individuals who struggle with identity by giving them the opportunity to improve or change personas. They use social networking to find psychological meaning to a deep and compelling need to feel emotionally close to others. In an online environment they are able to express themselves and find the acceptance missing in their lives.

Another pathway to IA may be body image problems and avoidance of real-life interactions because of embarrassment relating to one's appearance (Rodgers et al. 2013). IA can also result from compulsive consumption of online pornography due to its accessibility, affordability, and anonymity (Southern 2008).

\section{Conceptual diagnostic models for IA}

In line with the different approaches towards defining IA terminology, there are varying definitions and conceptualizations for IA diagnosis. Researchers view IA on a specter from a poorly controlled urge that causes distress to a serious psychopathology. This might be influenced by the prevalence and ubiquity of the Internet prevents the detection and diagnosis of IA, especially as its addictive patterns are masked by its legitimate everyday use for work or pleasure (Young 2009).

\section{Psychopathological models}

Research efforts have conceptualized at least three different models for which view IA as an obsessivecompulsive disorder (Grant et al. 2010), an impulse control disorder (Beard \& Wolf 2001) and/or an addictive disorder (Fisoun et al. 2012). 
OCD is represented by an intrusive anxiety to repetitively perform the same action. In an Internet environment the compulsive aspects of OCD can be observed with the urge to constantly participate in repetitive online activities (ex. check an email account, or a personal Facebook feed). Some pharmacological treatment studies which use selective serotonin reuptake inhibitors support the foundation for this model (Dell'Osso et al. 2006). In addition, it has been suggested that Internet addiction may be seen as the consequence of an obsessive passion defined as "a strong inclination towards a controlled internalization of an activity that a person likes, finds important, and in which both time and energy are invested, into one's identity" (Lafrenière et al. 2009, Przybylski et al. 2009). Such internalization is caused by "pressure" from the contingencies which are attached to the activity or by the uncontrollable sense of excitement derived from participation in the activity. Furthermore, the activity itself takes up a disproportionate amount of space in the person's identity and causes conflicts with other activities in the person's life.

Impulse-control disorders are characterized as an impaired inhibition to engage in repetitive behaviors that may also include adverse consequences (Aboujaoude 2010). IA has been described as impulsive, with individuals reporting an increased sense of tension or arousal before connecting to the Internet, and a relief of that tension once they were online (Shapira et al. 2000). Specifically, studies have shown that both lack of perseverance and urgency are positively related to Internet addiction as facets of impulsivity (Mottram \& Fleming 2009, Billieux et al. 2010). Recently, this classification has been viewed as too restrictive considering that other factors besides impulsivity traits also influence IA (Billieux et al. 2013). These factors include, but are not limited to personality traits, motives for Internet use, comorbid psychopathology, and specific Internet activity.

The addictive disorder approach conceptualizes IA as a "behavioral addiction" similar to a substance use disorder (Han et al. 2009). Results from neuroimaging experiments have shown neurobiological changes similar to those observed in substance addictions (Yuan et al. 2011). These changes include: lower activation in conflict detection, less efficiency in information processing, lower cognitive control, impaired executive control and enhanced reward sensitivity. "Addiction" is also suggested by the presence of symptoms which share similarities to substance addictions on both behavioral and biological aspects (Chakraborty et al. 2010), four of which are considered as highly relevant to any DSM-5 definition.

- Excessive use, which is often associated with a loss of a sense of time or a neglect of basic drives;

- Withdrawal, including feelings of anger, tension, and/ or depression, when the computer is inaccessible;

- Tolerance, including the need for better computer equipment, more software, or more hours of use; and

- Negative repercussions, including arguments, lying, poor achievement, social isolation, and fatigue.
Considering that the diagnostic criteria for impulse control disorders overlap with those for addictive disorders (Brewer \& Potenza 2008), IA has also been conceptualized as a combination of both. Based on clinical experience validated on a group of 405 subjects, Tao et al. (2010) expanded the four addictive disorder criteria, listed above, to a more complete IA diagnostic model. Their diagnostic definition, emphasizes the obligatory presence of two symptom criteria:

- Preoccupation with the Internet when offline;

- Withdrawal, manifested by dysphoric mood, anxiety, irritability, or boredom after being offline for several days.

Additionally, one or more of the following five symptom criteria is also present:

- Tolerance, defined as increased Internet use required to achieve the same satisfaction level;

- Persistent desire and/or unsuccessful attempts to control, cut back, or discontinue Internet use;

- Continued excessive use despite physical or psychological problems likely to have been caused or exacerbated by Internet use;

- Loss of previous interests, hobbies, and other pleasurable pursuits;

- Use of the Internet to escape or relieve a dysphoric mood.

And finally, combining the symptom criteria with the following three additional criteria increased the diagnostic accuracy to $99.26 \%$ with $89.66 \%$ specificity and $100 \%$ reliability.

- Excessive Internet use is not better accounted for by another disorder;

- Excessive Internet use results in functional impairment, such as academically, professionally, or in personal relationships;

- Duration of heavy Internet use exceeds 3 months, with at least 6 hours of Internet usage (non-business/non-academic) daily.

\section{Non-psychopathological interpretations}

Defining IA as a discrete psychopathological disorder has been a matter of debate. Most of the existing diagnostic definitions and scales often approach IA as a homogenous activity without consideration for the nature of the specific online activities. Their focus is commonly on the total time spent online and/or overall dysfunction, whereas the user's exact online pursuits are often detrimental for any comprehensive diagnosis. A total offline existence is no longer a realistic expectation, hence for a proper analysis it is necessary to assess the type of online activities against the amount of expected online presence due to education, work and/or leisure in order to determine the "healthy" level of online life.

Excessive Internet use is also viewed as a compensatory strategy rather than a genuine addictive behavior (Schimmenti \& Caretti 2010), making IA a mani- 
festation of an underlying disorder. Kardefelt-Winther (2014) proposes a theory of compensatory Internet use where negative life situations give rise to a motivation to go online to alleviate negative feelings. The basic tenet of this theory is that increased Internet use is a reaction by the individual to a negative life situation. For example, a lack of social stimulation would motivate the individual to go online to socialize via a social networking site or an online game. This action could have positive consequences as the individual compensates for the desired social stimulation in an understandable and practical way (Chappell et al. 2006), and negative consequences due to the amount of compensation required to alleviate negative feelings. Generally, the need for compensation may be constant for individuals with permanent real life issues, such as physical handicaps or disabilities (Griffiths 2000) while for less severe cases a few hours of compensatory internet use may be beneficial and lead to fewer problematic outcomes (Leung 2007).

IA often appears alongside numerous conditions that raise the questions of causality. Based on the available data on prognosis, stability, and treatment, classifying IA as an independent diagnosis of a mental disorder is considered premature, which was ultimately the reasoning behind its exclusion from the fifth edition of the Diagnostic and Statistical Manual of Mental Disorders (Pies 2009). Hence, there are still no clear guidance on how to approach or treat people with suspected Internet-related psychopathology or even how pursue research related to IA. Instead, both clinicians and researchers rely on definitions and screening and assessment instruments developed by the academic community. Finally, at the Internet is becoming a ubiquitous part of everyday life some of the symptoms and behaviors associated with Internet addiction can actually be interpreted as shift in how younger generations entertain or communicate with each other. As suggested by Smahel et al. (2008), what is treated by researchers as pathological behavior may be a new way of life for which researchers currently have only pathological interpretations.

\section{Comorbidity}

The comorbidity of two disorders may indicate a causal relationship or common etiology (Mueser et al. 1998). To shed light on the mechanism of IA, several comorbidity studies for psychiatric symptoms reveal which psychiatric symptoms are potentially associated with the development or maintenance of Internet addiction. The studies show that depression is repeatedly associated with Internet addiction for adolescents (Ha et al. 2006, Kim et al. 2006). Attention deficit hyperactivity disorder (ADHD), one of the most common psychiatric disorders, diagnosed has been reported to be associated with Internet addiction in children (Yoo et al. 2004). Nevertheless, the causal relationship between Internet addiction and ADHD in adolescents has not been directly evaluated. Furthermore, social phobia has been positively associated with Internet use in adolescents (Sheperd \& Edelmann 2005) yet the direct association between social phobia and Internet addiction has not been addressed.

There is no direct evidence for the impact of excessive Internet use on children's brain. As for behavior, research indicates that there is a complex relationship between Internet addiction and other emotional, social and psychological issues faced by the small percentage of children who might be classified as excessive users of the internet. These children tend to be older, to have emotional problems and to be 'sensation-seeking' (Green et al. 2012).

\section{TREATMENT}

While IA is not an officially recognized disorder, its adverse effects on health and functioning has initiated an area of research focused on treatment and prevention of IA. Despite their chemical or behavioral background, all addictions share specific characteristics and hence clinical interventions for IA are based on therapeutic and pharmacological strategies that are commonly used in previously established conditions: OCD, impulse control disorders and substance use disorders. The treatment itself consists of psychotherapy, pharmacology or a combination of both, as these types of interventions were shown to be highly effective for decreasing the amount of time Internet addicts spent online, and/or good at targeting symptoms of depression and anxiety (Winkler et al. 2013). Nevertheless, published studies on this topic are scarce, precluding any recommendations for strong treatments. Even most of the existing studies have been found to be inconsistent with the definition and diagnosis of IA, lack randomization and comparison groups and provide insufficient information about recruitment protocols (King et al. 2011).

\section{Psychological Treatment}

The mainstream psychological treatment of IA is focused around cognitive-behavioral therapy (CBT), considered as the most influential model (Young, 2013). The therapy requires approximately 12 weekly session where the early stages are focused on the behavioral aspects of the patient, while in subsequent stages the treatment is gradually shifted towards confronting the cognitive assumptions and distortions that encourage the problematic behavior. During treatment Internet addicts identify affective and situational triggers associated with their addictive online behavior and learn how to modify them into more adaptive ones (Khazaal et al. 2012). In addition, CBT encourages the stimulation of activities which do not involve the use of the Internet in order to treat specific comorbidities. For example, the decreased use of the Internet decreases the dopamine levels in the individual. Participation in physical activities would compensate for this decrease in dopamine and enhance 
the effectiveness of the intervention (Cash et al. 2012). Most participants in CBT therapy manage to deal with their complaints by the eight session, with maintained improvement at a 6-month follow-up (Young 2007).

In a randomized study of 56 Chinese adolescents divided into active treatment and waitlist control groups, only the active treatment groups demonstrated an improvement in time management skills and a decrease in emotional, cognitive, and behavioral symptoms post intervention (Du et al. 2010). Participants in the active treatment group were treated with a multimodal group CBT which included effective communication with parents, management of online relationships, and techniques for recognizing and controlling problematic impulses. Zhu et al. (2012) describe a study which combines psychological intervention on cognitive function and event-related potentials with electro-acupuncture as a treatment for patients with IA. Participants from all groups had significantly lower IA scores while scores of short-term memory capacity and short-term memory span increased significantly.

Specific variations of cognitive-behavioral therapy have also been devised to treat IA, such as CBT-IA (Young 2011). Nevertheless, although this model appears to be effective, there seem to be no significant differences between this type of treatment and other psychological approaches. Conversely, other psychological treatments that might be beneficial are selfimposed bans on Internet access (Shaw \& Black 2008), abstinence programs (Kalke \& Raschke 2004), counseling programs (Shek et al. 2009) or multifamily group therapy (Liu et al. 2015). Considering that IA is a complex behavioral disorder it is necessary to develop a wide range of interventions that will suit the various needs and conditions of the individuals which are experiencing difficulties. In the field of IA, different treatment methods should be developed and their effectiveness should be evaluated in settings including individual and group psychotherapy. In addition, group work treatments could be effective for people with IA disorders. For example, groups for personal change are oriented on the transformation of dysfunctional behavior of members or psychoeducational groups with a dual focus on using information and educational processes to facilitate individual grow (Ajduković 1997, Meath 2016). Group treatment of IA disorder presents an opportunity for multidisciplinary interventions which would include medical professionals, social workers and psychologists. Current practice in psychiatric institutions offers day hospitals where psychotherapy is combined with group work and relaxation techniques, occupational therapy as well as mandatory group multifamily therapy.

It is important to emphasize that in the treatment of IA abstinence is rarely possible because the Internet is used for a wide range of professional and educational activities in everyday life. IA could be the first addiction where abstinence is not preferable and focus in treatment should move from abstinence to responsible use of internet in work and leisure time.

\section{Pharmacological Treatment}

As mentioned previously, pharmacological studies of IA utilize drugs that are extrapolated from treatments of established conditions for OCD, impulse control and substance use disorders.

Based on the shared features between IA and OCD, a study of escitalopram, an SSRI effective in OCD, was conducted on 19 adults (Dell'Osso et al. 2006). Although there was a significant decrease in time spent online in the open-label phase of the treatment, in the randomized phase both treatment and placebo groups performed within the same parameters. Extendedrelease methylphenidate, a central nervous system stimulant used in ADHD treatment, was tested on 62 South Korean children who were addicted to Internet gaming (Han et al. 2009). The results showed a significant decrease in time spent online, which was in turn positively correlated to improvement in attention, possibly due to the regulation of dopamine levels. Bupropion, an inhibitor medication used in the treatment of substance dependence was used for the treatment of Internet videogame addiction (Han et al. 2010). After 6 weeks, the craving for gameplay and total play time was significantly reduced. A follow-up study, showed that besides total play time, bupropion, an antidepressant medication, also reduced Internet addiction and Beck Depression Inventory scores in a group of excessive video game players (Han \& Renshaw 2012).

Other isolated studies have used naltrexone, a medication that stops opioid activity, commonly used for impulse control disorders, to treat compulsive cybersexual behavior (Bostwick \& Bucci 2008) or a combination of atypical antipsychotic quetiapine with to citalopram (an SSRI) to treat IA (Atmaca 2007).

Both psychological and pharmacological interventions have been effective in treating and reducing IA symptoms. Based on the existing evidence, clinicians should combine both approaches to treat this phenomenon because as a strategy it has been found to be the most effective method for treating IA (Przepiorka et al. 2014). Total abstinence from the Internet should not be the goal of any intervention, and that instead an abstinence from problematic online activities and regulated use of the activity should be achieved (Cash et al. 2012). Due to the specificity of IA it is important to assess the nature of excessive Internet use and how it relates to patients' online behavior in general in order to gain a better insight into motivations underpinning excessive and harmful use.

\section{CONCLUSION}

Internet addiction is complex construct, actual in the focus of experts in multidisciplinary environment. Development of various treatment practices demonstrates the need for a multidisciplinary approach involving various professions from medical sector as well 
as social workers and psychologists. The symptomatology of internet addictive behavior and its diagnosis model is particularly important for health workers, especially those working with children and youth. The recent trend of growing internet use shows need for intervention for efficient intervention of professionals.

The past two decades show efforts of researches in the field regarding conceptualization and operationalization of the problematic internet use. Currently authors and researchers still use different definitions in order to operationalize the IA disorder hence it is feasible to conclude that there is no consensus on the conceptualization which limits the comparison results. Although current literature presents useful findings in the field of internet addiction future researches in the field should contribute to explanation of online risk behaviors and effective treatment. In addition, the observed difference in dimensional structures when using identical or dissimilar instruments, indicate that the observed variables do not conform to a single model. While this has only been researched in terms of culture, it implies that either the instrument or the observed phenomenon, IA, is not consistent. This further prevents quantifiable measurement and classification of internet addiction and needs to be addressed in future research.

Prevention measures should be planned on all three levels of evidence based programs. Universal prevention should be directed to the general population without prior assessment with the aim of informing and educating the entire population and acquiring skills for responsible internet use through educational school programs from early age in IT and media education. Selective prevention programs should be targeted at population groups that show an increased degree of risk in relation to the general population. For the quality planning and implementation of selective prevention programs, research should be carried out to identify risk groups. Future research should explore risk factors for the occurrence of IA disorder ensuring evidence based practice in prevention programs. Indicated prevention programs are aimed at preventing or reducing further occurrence of difficulties and are directed towards those individuals who already identified a certain level of disorder. This usually requires more intensive and long-lasting programs conducted by educated professionals (National Research Council 2009). Carefully planned evaluation of prevention and treatment programs are important and can give significant feedback on effectiveness of interventions.

Online activities have become an integral part of our everyday life. It is impossible to avoid them as our virtual self is an integral part of our real life and personal identity. Considering how quickly modern technology develops, efficient and timely intervention is needed in the field of regulation policies, prevention, identification and treatment of internet addictive behaviors.

\section{Acknowledgements: None.}

Conflict of interest: None to declare.

\section{Contribution of individual authors:}

Martin Mihajlov: design of the review, literature search (data collection), writing the article, critical revision, final approval.

Lucija Vejmelka: literature search (data collection), writing the article, critical revision.

\section{References}

1. Aa $N$ Van der, Overbeek $G$ \& Engels RCME: Daily and Compulsive Internet Use and Well-Being in Adolescence: A Diathesis-Stress Model Based on Big Five Personality Traits Daily Internet Use and Low Well-Being. J Youth Adolesc 2008; 1-19.

2. Aboujaoude E, Koran LM, Gamel N, Large MD \& Serpe RT: Potential markers for problematic internet use: A telephone survey of 2,513 adults. CNS Spectr 2006; 11:750-755.

3. Aboujaoude E: Problematic Internet use: an overview. World Psychiatry, 2010;

4. Ajduković M: Grupni pristup u psihosocijalnom radu: načela i procesi, 1997;

5. Alavi SS, Fereshte J, Mehdi E \& Hossein R: Validity, reliability and factor analysis of compulsive internet use scale in students of Isfahan's universities. Heal Inf Manag J 2011; 7:715-724.

6. American Psychiatric Association: Diagnostic and statistical manual of mental disorders (5th ed.), 2013;

7. Atmaca M: A case of problematic internet use successfully treated with an SSRI-antipsychotic combination. Prog Neuro-Psychopharmacology Biol Psychiatry 2007; 31:961-962.

8. Bakken IJ, Wenzel HG, Götestam KG, Johansson A \& Øren A: Internet addiction among Norwegian adults: A stratified probability sample study. Scand J Psychol 2009; 50:121-127.

9. Barke A, Nyenhuis $N \&$ Kroner-Herwig B: The German version of the internet addiction test: a validation study. Cyberpsychol Behav Soc Netw 2012; 15:534-542.

10. Beard KW \& Wolf EM: Modification in the proposed diagnostic criteria for Internet addiction. Cyberpsychology Behav 2001; 4:377-383.

11. Beard KW: Internet addiction: a review of current assessment techniques and potential assessment questions. CyberPsychology Behav 2005; 8:7-14.

12. Bener A \& Bhugra D: Lifestyle and depressive risk factors associated with problematic internet use in adolescents in an Arabian Gulf culture. J Addict Med 2013; 7:236-242.

13. Billieux J, Gay P, Rochat L \& Linden M Van der: The role of urgency and its underlying psychological mechanisms in problematic behaviours.pdf. Behav Res 2010;

14. Billieux J, Van Der Linden M, Achab S, Khazaal Y, Paraskevopoulos L, Zullino D \& Thorens G: Why do you play World of Warcraft? An in-depth exploration of selfreported motivations to play online and in-game behaviours in the virtual world of Azeroth. Comput Human Behav 2013; 29:103-109. 
15. Billieux J: Problematic Use of the Mobile Phone: A Literature Review and a Pathways Model. Curr Psychiatry Rev 2012; 8:299-307.

16. Black DW, Belsare G, Schlosser S. Clinical Features, Psychiatric Comorbidity \& Health-Related Quality of Life in Persons Reporting Compulsive Computer Use Behavior: J Clin Psychiatry 1999; 60:839-844.

17. Block JJ: Issues for DSM V: Internet Addiction. Am J Psychiatry 2008; 165:306-307.

18. Bostwick JM \& Bucci JA: Internet Sex Addiction Treated with Naltrexone. Mayo Clin Proc@BULLET Febr 2008; 83:226-230.

19. Brewer JA \& Potenza MN: The neurobiology and genetics of impulse control disorders: Relationships to drug addictions. Biochem Pharmacol 2008; 75:63-75.

20. Cantelmi T, Del Miglio C, Talli $M$ \& D’Andrea A: Internetrelated psychopathology: first experimental data, clinical aspects and critical notes. Ital J Psychopathol 2000; 6:40-51.

21. Caplan S: Problematic internet use and psychosocial well being: Development of a theory-based cognitive behavioural measurement instrument. Comput Hum Behav 2002; 18:553-575.

22. Cartierre N, Coulon $N \&$ Demerval R: Validation d'une version courte en langue francaise pour adolescents de la Compulsive Internet Use Scale. Neuropsychiatr Enfance Adolesc 2011; 59:415-419.

23. Cash H, D. Rae C, H. Steel A \& Winkler A: Internet Addiction: A Brief Summary of Research and Practice. Curr Psychiatry Rev 2012; 8:292-298.

24. Chakraborty K, Basu D \& Kumar KGV: Internet addiction: consensus, controversies, and the way ahead. East Asian Arch Psychiatry 2010; 20:123.

25. Chappell D, Eatough V, Davies MNO \& Griffiths M: Ever Quest - It's Just a Computer Game Right? An Interpretative Phenomenological Analysis of Online Gaming Addiction. Int J Ment Health Addict 2006; 4:205-216.

26. Charlton JP \& Danforth IDW: Distinguishing addiction and high engagement in the context of online game playing. Comput Human Behav 2007; 23:1531-1548.

27. Chen $S$, Weng L, Su Y, Wu H \& Yang P: Development of a Chinese Internet addiction scale and its psychometric study. Chinese J Psychol 2003; 45:279.

28. Council NR: Preventing mental, emotional, and behavioral disorders among young people: Progress and possibilities, 2009;

29. Davis R, Flett GL \& Besser A: Validation of a new scale for measuring problematic internet use: implications for pre-employment screening. Cyberpsychol Behav 2002; 5:331-345.

30. Davis RA: Cognitive-behavioral model of pathological Internet use. Comput Human Behav 2001; 17:187-195.

31. Dell'Osso B, Altamura AC, Hadley SJ, Baker BR \& Hollander E: An open-label trial of escitalopram in the treatment of impulsive-compulsive internet usage disorder. Eur Neuropsychopharmacol 2006; 16:S82-S83.

32. Demetrovics Z, Szeredi B \& Rózsa S: The three-factor model of Internet addiction: the development of the Problematic Internet Use Questionnaire. Behav Res Methods 2008; 40:563-574.

33. Deng YX, Hu M, Hu GQ, Wang LS \& Sun ZQ: [An investigation on the prevalence of internet addiction disorder in middle school students of Hunan province]. Zhonghua liu xing bing xue za zhi= Zhonghua liuxingbingxue zazhi 2007; 28:445-448.
34. Dhir A \& Helsinki PO: On the Nature of Internet Addiction. What is it and how is it measured? 978-951-511119-7 2015,

35. Du YS, Jiang $W$ \& Vance A: Longer term effect of randomized, controlled group cognitive behavioural therapy for Internet addiction in adolescent students in Shanghai. 2010; 44:129-134.

36. Durkee T, Kaess M, Carli V, Brunner $R \&$ Wasserman D: P-598 - Prevalence of pathological internet use among adolescents in Europe: demographic and health-related risk factors. Eur Psychiatry 2012; 27(Suppl 1).

37. Eijnden R Van den \& Meerkerk G: Online communication, compulsive Internet use, and psychosocial well-being among adolescents: a longitudinal study. Developmental 2008;

38. Fioravanti G, Primi $C$ \& Casale S: Psychometric evaluation of the Generalized Problematic Internet Use Scale 2 in an Italian sample. Cyberpsychol Behav Soc Netw 2013; 16:761-6.

39. Fisoun V, Floros $G$, Siomos K, Geroukalis D \& Navridis $K$ : Internet addiction as an important predictor in early detection of adolescent drug use experience-implications for research and practice. J Addict Med 2012; 6:77-84.

40. Gámez-Guadix M, Villa-George FI \& Calvete E: Measurement and analysis of the cognitive-behavioral model of generalized problematic Internet use among Mexican adolescents. J Adolesc 2012; 35:1581-1591.

41. Goel D, Subramanyam A \& Kamath R: A study on the prevalence of internet addiction and its association with psychopathology in Indian adolescents. Indian $J$ Psychiatry 2013; 55:140-3.

42. Grant JE, Potenza MN, Weinstein A \& Gorelick DA. Introduction to behavioral addictions. Am J Drug Alcohol Abus 2010; 36: 233-241.

43. Griffiths $M D \&$ Pontes HM: Internet addiction disorder and internet gaming disorder are not the same. J Addict Res Ther 2014; 5:e124.

44. Griffiths MD: Does Internet and Computer 'Addiction' Exist? Some Case Study Evidence. CyberPsychology Behav 2000; 3:211-218.

45. Griffiths MD: Gambling on the Internet: A brief note. Journal of Gambling Studies. J Gambl Stud 1996; 12: 471-473.

46. Griffiths MD: Internet addiction: Internet fuels other addictions. Student Br Med J 1999; 7:428-429.

47. Griffiths MD: Internet ' addiction': an issue for clinical psychology? Clin Psychol Forum 1996; 97:32-36.

48. Griffiths MD: Technological addictions. Clin Psychol Forum 1995; 76:14-19.

49. Guan SSA \& Subrahmanyam K: Youth Internet use: risks and opportunities. Curr Opin Psychiatry 2009; 22:351356.

50. Guertler D, Rumpf HJ, Bischof A, Kastirke N, Petersen $K U$, John $U$ \& Meyer C: Assessment of problematic internet use by the compulsive internet use scale and the internet addiction test: A sample of problematic and pathological gamblers. Eur Addict Res 2014; 20: 75-81.

51. Ha JH, Yoo HJ, Cho IH, et al.: Psychiatric comorbidity assessed in Korean children and adolescents who screen positive for Internet addiction. J Clin Psychiatry 2006; 67:821-6.

52. Hall A \& Parsons J: Internet addiction: College student case study using best practices in cognitive behavior therapy. J Ment Heal Couns 2001; 23:312-327. 
53. Han D\& Renshaw P: Bupropion in the treatment of problematic online game play in patients with major depressive disorder. J Psychopharmacol 2012;

54. Han D, Lee Y, Na C, Ahn J, Chung U, Daniels MA, Haws $C A \&$ Renshaw PF: The effect of methylphenidate on Internet video game play in children with attentiondeficit/hyperactivity disorder. Compr Psychiatry 2009; 50:251-256.

55. Han DH, Hwang JW \& Renshaw PF: Bupropion sustained release treatment decreases craving for video games and cue-induced brain activity in patients with Internet video game addiction. Exp Clin Psychopharmacol 2010, 18:297-304.

56. Internet Live Stats: Internet Users. http://www.internetlivestats.com/internet-users/.

57. Jr DH: Pornography addiction-a supranormal stimulus considered in the context of neuroplasticity. Socioaffective Neurosci Psychol 2013;

58. Kalke $J$ \& Raschke P: Learning by doing: 'Initiated abstinence', a school-based programme for the prevention of addiction: Results of an evaluation study. Eur Addict Res 2004; 10:88-94.

59. Kanizaj I: EU Kids Online. 2016,

60. Karaiskos D, Tzavellas E \& Balta G: P02-232-Social network addiction: a new clinical disorder? Eur Psychiatry 2010;

61. Kardefelt-winther D: Computers in Human Behavior A conceptual and methodological critique of internet addiction research: Towards a model of compensatory internet use. Comput Human Behav 2014; 31:351-354.

62. Kesici $S \&$ Sahin I: Turkish adaptation study of Internet Addiction Scale. Cyberpsychol Behav Soc Netw 2010; 13:185-189.

63. Khazaal Y, Chatton A \& Atwi K: Arabic validation of the compulsive Internet use scale (CIUS). Substance 2011,

64. Khazaal Y, Xirossavidou C, Khan R \& Edel Y: Cognitivebehavioral treatments for internet addiction. Open Addict 2012;

65. Kim K, Ryu E, Chon MY, Yeun EJ, Choi SY \& Seo JS. Internet addiction in Korean adolescents and its relation to depression and suicidal ideation: a questionnaire survey. Int J Nurs Stud 2006; 43:185-192.

66. Kimberly S: Young $C N$ de A. Internet Addiction: A Handbook and Guide to Evaluation and Treatment, 2010;

67. King D, Delfabbro $P \&$ Griffiths M: Assessing clinical trials of Internet addiction treatment: A systematic review and CONSORT evaluation. Clin Psychol Rev 2011;

68. Király O, Griffiths MD, Urbán R, Farkas J, Kökönyei G, Elekes Z, Tamás D \& Demetrovics Z: Problematic internet use and problematic online gaming are not the same: findings from a large nationally representative adolescent sample. Cyberpsychology, Behav Soc Netw 2014; 17:749_ 754.

69. Ko CH, Yen JY, Yen CF, Chen CC, Yen CN \& Chen SH: Screening for Internet addiction: an empirical study on cut-off points for the Chen Internet Addiction Scale. Kaohsiung J Med Sci 2005; 21:545-51.

70. Kuss DJ, Griffiths MD, Karila L \& Billieux J: Internet addiction: A systematic review of epidemiological research for the last decade. Curr Pharm Des 2014; 20:1-27.

71. Laconi S, Rodgers RF \& Chabrol H: The measurement of Internet addiction: a critical review of existing scales and their psychometric properties. Comput Human Behav 2014: 41:190-202.
72. Lafrenière MAAK, Vallerand RJ, Donahue EG, Lavigne GL \& Lafreniere MA: On the costs and benefits of gaming: the role of passion. Cyberpsychol Behav 2009; 12:285-290.

73. Lee BW \& Stapinski LA: Journal of Anxiety Disorders Seeking safety on the internet: Relationship between social anxiety and problematic internet use. J Anxiety Disord 2012; 26:197-205

74. Lee K, Gyeong H, Yu B, Song YM, Lee HK \& Kim D: Reliability and validity of the Korean version of the internet addiction test among college students. $J$ Korean Med Sci 2013; 28:763-768.

75. Lemenager T, Gwodz A, Richter A \& Reinhard I: Selfconcept deficits in massively multiplayer online roleplaying games addiction. Eur Addict 2013;

76. Leung L: Stressful life events, motives for internet use, and social support among digital kids. Cyberpsychology Behav 2007; 10.

77. Li D, Liau A \& Khoo A: Examining the influence of actual-ideal self-discrepancies, depression, and escapism, on pathological gaming among massively multiplayer online adolescent gamers. Cyberpsychol Behav Soc Netw 2011; 14:535-539.

78. Liu QX, Fang XY, Yan N, Zhou ZK, Yuan XJ, Lan J \& Liu $C Y$ : Multi-family group therapy for adolescent Internet addiction: Exploring the underlying mechanisms. Addict Behav 2015; 42:1-8.

79. Lortie CL \& Guitton MJ: Internet addiction assessment tools: Dimensional structure and methodological status. Addiction 2013; 108:1207-1216.

80. Love $T$, Laier $C$, Brand $M$, Hatch $L$ \& Hajela $R$ Neuroscience of internet pornography addiction: a review and update. Behav Sci (Basel) 2015;

81. Marks I: Behavioural (nonchemical) addictions. Br J Addict 1990;

82. Meath S: An Experiential Approach to Group Work. Sch Soc Work J 2016;

83. Meerkerk G, Van Den Eijnden RJJM, Vermulst AA \& Garretsen HFL: The Compulsive Internet Use Scale (CIUS): some psychometric properties. Cyberpsychol Behav 2009; 12:1-6.

84. Morahan MJ: Incidence and correlates of pathologic internet use among college students. Comput HumBehav 2000; 16:13-29.

85. Moreno MA, Jelenchick L, Cox E, Young H \& Christakis DA: Problematic internet use among US youth: a systematic review. Arch Pediatr Adolesc Med 2011;165: 797-805.

86. Mottram AJ \& Fleming MJ: Extraversion, impulsivity, and online group membership as predictors of problematic internet use. Cyberpsychol Behav 2009; 12: 319-321.

87. Mueser KT, Drake RE \& Wallach MA: Dual diagnosis: a review of etiological theories. Addict Behav 1998; 23:717-34.

88. Osada H: Internet addiction in Japanese college students: Is Japanese version of Internet Addiction Test (JIAT) useful as a screening tool? Bull Senshu Univ Sch Hum Sci 2013; 3:71-80.

89. Ozcan NK \& Buzlu S: An assistive tool in determining problematic internet use: validity and reliability of the Online Cognition Scale in a sample of university students. $J$ Depend 2005; 6:19-26.

90. Petersen KU, Weymann N, Schelb $Y$, Thiel $R \&$ Thomasius $R$ : [Pathological Internet use - epidemiology, diagnostics, 
co-occurring disorders and treatment]. Fortschr Neurol Psychiatr 2009; 77:263-271.

91. Petry N, Rehbein F, Ko C \& O'Brien C: Internet gaming disorder in the DSM-5. Curr Psychiatry Rep 2015;

92. Peukert P, Steffen S, ElKasmi J, Barth GM, Meerkerk GJ \& Batra A: Faktorielle Struktur der Deutschen version der Compulsive Internet Use Scale (CIUS) nach konfirmatorischer Faktorenanalyse. Z Klin Psychol Psychother 2012; 41:101-108.

93. Pezoa-Jares $R$ \& Espinoza-Luna I: Internet addiction: A review. J Addict 2013,

94. Pies R: Should DSM-V Designate 'Internet Addiction' a Mental Disorder? Psychiatry (Edgmont) 2009; 6:31-37.

95. Pontes H, Szabo A \& Griffiths M: The impact of Internetbased specific activities on the perceptions of Internet addiction, quality of life, and excessive usage: A crosssectional study. Addict Behav Reports 2015;

96. Pratarelli ME \& Browne BL: Confirmatory factor analysis of Internet use and addiction. CyberPsychology Behav 2002; 5: 53-64.

97. Pratarelli ME, Browne BL \& Johnson K: The bits and bytes of computer/Internet addiction: A factor analytic approach. Behav Res Methods, Instruments, Comput 1999; 31:305-314.

98. Przepiorka AM, Blachnio A, Miziak B \& Czuczwar SJ: Clinical approaches to treatment of Internet addiction. Pharmacol Reports 2014; 66:187-191.

99. Przybylski AK, Weinstein N, Ryan RM \& Rigby CS: Having to versus wanting to play: background and consequences of harmonious versus obsessive engagement in video games. Cyberpsychology Behav impact Internet, Multimed virtual Real Behav Soc 2009; 12:485-92.

100. Ramezani $M$, Salehi M, Namiranian $N \&$ Salehi $M$ : Validity and reliability of the Chen internet addiction scale. J Fundam Ment Heal 2012; 14: 236-245.

101. Recupero PR: The Mental Status Examination in the Age of the Internet. J Am Acad Psychiatry 2010; 38.

102. Rodgers RF, Melioli T, Laconi S, Bui E \& Chabrol H: Internet addiction symptoms, disordered eating, and body image avoidance. Cyberpsychol Behav Soc Netw 2013; 16:56-60.

103. Rumpf HJ, Vermulst AA, Bischof A, Kastirke N, Gurtler $D$, Bischof G, Meerkerk GJ, John $U$ \& Meyer $C$ : Occurence of internet addiction in a general population sample: a latent class analysis. Eur Addict Res 2014; 20:159-166.

104. Saville BK, Gisbert A, Kopp J \& Telesco C: Internet addiction and delay discounting in college students. Psychol Rec 2010; 60:273-286.

105. Schimmenti A \& Caretti V: Psychic retreats or psychic pits?: Unbearable states of mind and technological addiction. Psychoanal Psychol 2010; 27:115-132.

106. Shapira NA, Goldsmith TD, Keck PE, Khosla UM \& McElroy SL: Psychiatric features of individuals with problematic internet use. J Affect Disord 2000; 57:267-272.

107. Shaw $M$ \& Black D: Internet addiction. CNS Drugs 2008;

108. Shek DTL, Tang VMY \& Lo CY: Evaluation of an Internet addiction treatment program for Chinese adolescents in Hong Kong. Adolescence 2009; 44:359373.

109. Shepherd RM \& Edelmann RJ: Reasons for internet use and social anxiety. Pers Individ Dif 2005; 39:949-58.
110. Smahel D, Blinka L \& Ledabyl O: Playing MMORPGs: connections between addiction and identifying with a character. Cyberpsychol Behav 2008; 11:715-718.

111. Smahel D, Brown BB \& Blinka L: Associations between online friendship and Internet addiction among adolescents and emerging adults. Dev Psychol 2012; 48:381-388.

112. Song J: The Usage of Online Cognitive Scale in 538 Medical Undergraduates. Chinese Ment Heal J 2007; 21:525.

113. Southern S: Treatment of Compulsive Cybersex Behavior. Psychiatr Clin North Am 2008; 31:697-712.

114. Spicer J, Galvan A, Hare T, Voss $H$ \& Glover G: Sensitivity of the nucleus accumbens to violations in expectation of reward. Neuroimage 2007;

115. Starcevic V: Is internet addiction a useful concept? Spec Issue Aust New Zeal J Psychiatry 2013; 47:16-19.

116. Stats IW: Internet users in Europe. 2017;

117. Tao HK: Teenagers' Internet addiction and the qualityoriented education. J High Corresp Educ (Philosophy Soc Sci 2005; 3:70-73.

118. Tao R, Huang $X$, Wang J, Zhang H, Zhang $Y \& L i M$ : Proposed diagnostic criteria for internet addiction. Addiction 2010; 105:556-564.

119. teWildt BT: Internet dependency: Symptoms, diagnosis and therapy. Virtual worlds Crim 2011,

120. Tonioni F: Psicopatologia web-mediata. Psicopatol web-mediata 2013;

121. Tsitsika A \& Janikian M: Internet addictive behavior in adolescence: a cross-sectional study in seven European countries. Cyberpsychology, 2014;

122. Turel $O$ \& Serenko A: Is Mobile Email Addiction Overlooked? Commun ACM 2010; 53: 41-43.

123. Turel O, Serenko A \& Giles P: Integrating Technology Addiction and Use: an Empirical Investigation of Online Auction Users. MIS Q 2011; 35:1043-A18.

124. Vejmelka L, Strabic $N$ \& Jazvo M: Online aktivnosti $i$ rizična ponašanja adolescenata $u$ virtualnom okruženju. Društvena istraživanja 2017;

125. Wartberg L, Petersen KU, Kammerl R, Rosenkranz $M$ \& Thomasius R: Psychometric validation of a German version of the compulsive Internet use scale. Cyberpsychology, Behav Soc Netw 2014;17: 99-103.

126. Weinstein $A$ \& Lejoyeux $M$ : Internet addiction or excessive internet use. Am J Drug Alcohol Abuse 2010; 36:277-283.

127. Widyanto L, Griffiths MD \& Brunsden $V: A$ psychometric comparison of the Internet Addiction Test, the Internet-Related Problem Scale, and self-diagnosis. Cyberpsychol Behav Soc Netw 2011; 14:141-149.

128. Winkler A, Dorsing B, Rief W, Shen $Y \&$ Glombiewski JA: Treatment of internet addiction: A meta-analysis. Clin Psychol Rev 2013; 33:317-329.

129. Wu X, Chen X, Han J, Meng H, Luo J, Nydegger L \& Wu $H$ : Prevalence and Factors of Addictive Internet Use among Adolescents in Wuhan, China: Interactions of Parental Relationship with Age and HyperactivityImpulsivity. PLoS One 2013; 8.

130. Yoo HJ, Cho SC, Ha J, et al.: Attention deficit hyperactivity symptoms and internet addiction. Psychiatry Clin Neurosci 2004; 58:487-94.

131. Young K: Internet Addicition: Diagnosis and Treatment Considerations. Contemp Psychother 2009; 39:241246. 
132. Young K: Psychology of computer use: XL. Addictive use of the Internet: A case that breaks the stereotype. Psychol Rep 1996; 79:899-902.

133. Young KS, Pistner M, O'Mara J \& Buchanan J: Cyber disorders: The mental health concern for the new millennium. CyberPsychology Behav 1999; 2:475-479.

134. Young KS: Caught in the Net: How to recognize the signs of Internet addiction and a winning strategy for recovery. New York: Wiley, 1998b;

135. Young KS: CBT-IA: The First Treatment Model for Internet Addiction. J Cogn Psychother An Int Q 2011; 25:304-312.

136. Young KS: Cognitive behavior therapy with internet addicts: Treatment outcomes and implications. Cyberpsychology Behav 2007; 10:671-679.

137. Young KS: Internet Addiction: The Emergence of a New Clinical Disorder. CyberPsychology Behav 1998a; 1:237-244.
138. Young KS: Treatment outcomes using CBT-IA with Internet-addicted patients. J Behav Addict 2013; 2:209215.

139. Yuan K, Qin W, Liu Y \& Tian J: Internet addiction: Neuroimaging findings. Commun Integr Biol 2011; 4:637-639.

140. Zanetta Dauriat F, Zermatten A, Billieux J, Thorens G, Bondolfi $G$, Zullino D \& Khazaal Y: Motivations to play specifically predict excessive involvement in massively multiplayer online role-playing games: Evidence from an online survey. Eur Addict Res 2011; 17:185-189.

141. Zec G: Factor structure of the Online Cognition Scale and prediction of a pathological internet use, 2005;

142. Zhu TM, Li H, Jin RJ, Zheng Z, Luo Y, Ye H \& Zhu HM: Effects of electroacupuncture combined psycho-intervention on cognitive function and event-related potentials P300 and mismatch negativity in patients with internet addiction. Chin J Integr Med 2012; 18:146-151.

\section{Correspondence:}

Ass. Prof. Martin Mihajlov, MD

Cyril and Methodius University, Faculty of Economics

Goce Delcev 9b, Skopje, Macedonia,martin@eccf.ukim.edu.mk E-mail: martin@eccf.ukim.edu.mk 